\title{
SmGRAS5 acts as a positive regulator in GA-induced biosynthesis of tanshinones in Salvia miltiorrhiza hairy roots
}

\author{
Wenrui Li ${ }^{1}$, Dongfeng $\mathrm{Yang}^{2}$, Zhenqing $\mathrm{Bai}^{3}$, Tianlin $\mathrm{Pei}^{3}$, Renjun $\mathrm{Mao}^{3}$, Ruilian $\mathrm{Han}^{2}$, \\ and Zongsuo Liang ${ }^{1}$ \\ ${ }^{1}$ Institute of Soil and Water Conservation Chinese Academy of Sciences and Ministry of \\ Water Resources \\ ${ }^{2}$ Zhejiang Sci-Tech University \\ ${ }^{3}$ Northwest Agriculture and Forestry University
}

May 5, 2020

\begin{abstract}
GA is an important phytohormone that regulates root growth and secondary metabolism. GRAS family transcription factors (TFs) are the key regulators of GA signaling. Here, we found that SmGRAS5 was co-expressed in the root periderm with tanshinones in Salvia miltiorrhiza. Overexpression (OE) of SmGRAS5 increased tanshinones accumulation and upregulated the biosynthetic genes. Antisense expression (AE) of SmGRAS5 reduced tanshinones accumulation and downregulated the biosynthetic genes. Yeast one-hybrid (Y1H), dual-luciferase (Dual-LUC) and electrophoretic mobility shift assays (EMSA) showed that SmGRAS5 promoted tanshinones biosynthesis by directly binding to the GARE motif in the promoter of SmKSL1 to induce its expression. However, overexpressing SmGRAS5 reduced GA content through downregulating biosynthetic genes. GA treatment further increased tanshinones accumulation and restored the accumulation of GA inhibited by overexpressing SmGRAS5. SmGRAS5 could not directly bind to the GA biosynthetic genes. Transcriptome analysis revealed the potential functions of SmGRAS5 in regulating secondary metabolism. Taken together, SmGRAS5 regulated GA-promoted tanshinones biosynthesis by directly activating the expression of SmKSL1, which suggested that SmGRAS5 may be a potential target for further metabolic engineering of tanshinones biosynthesis in S. miltiorrhiza.
\end{abstract}

\section{INTRODUCTION}

The dried roots of Salvia miltiorrhiza Bunge (Danshen) are widely used in treating inflammation and heart diseases (Dong et al ., 2011; Liu et al ., 2018). S. miltiorrhiza has been considered as a model medicinal plant due to its important medicinal value, small genome ( $\sim 00 \mathrm{Mb})$, short life cycle, established transgenic system, and ease of tissue culture (Ma et al ., 2012; Xu et al ., 2015). The bioactive compounds of $S$. miltiorrhiza form two main groups: hydrophilic components (SAs), such as salvianolic acid B (Sal B) and rosmarinic acid (RA), and lipophilic components (tanshinones, which are a group of diterpenoids), such as dihydrotanshinone I (DT-I), cryptotanshinone (CT), tanshinone I (T-I) and tanshinone IIA (T-IIA) (Wang et al ., 2007; Pei et al ., 2018). The Chinese Pharmacopoeia stipulates that the main quality markers of Danshen are tanshinones and SAs (The State Pharmacopoeia Commission of China, 2015). The biosynthetic pathways of SAs include phenylpropanoid and tyrosine-derived pathways (Petersen, 2013; Sun et al. , 2018). Due to the great medicinal value of tanshinones, the biosynthetic pathways have been well studied (Ma et al ., 2015; Song et al. , 2015; Zhouet al., 2017). The tanshinones are synthesized from diterpenoids universal precursor GGPP, which is produced by the mevalonic acid (MVA) and 2-C-methyl-D-erythritol4-phosphate (MEP) pathways (Guo et al. , 2016; Pei et al. , 2018). The key enzyme genes involved in 
tanshinones biosyntheses, such as AACT, DXS , CMK , GGPPS , CPS , KSL1, and CYPr6AH1 have been characterized in $S$. miltiorrhiza (Ma et al ., 2012; Xuet al. , 2015). There have been many reports of overexpressing or RNA interference these biosynthetic genes could regulate the accumulation of tanshinones (Kai et al ., 2011; Ma et al ., 2016; Shi et al ., 2016). Overall, the supply of these bioactive compounds is limited because of their low concentrations in the roots of S. miltiorrhiza. And the yields and quality of $S$. miltiorrhiza are often affected by unfavorable environmental conditions. Therefore, research on regulating secondary metabolites has become a hot topic. The application of TFs is a promising approach to improve the efficiency of metabolic engineering in the plant (Fu et al. , 2018).

TFs play an important role in secondary metabolic engineering. TF can coordinately regulate the expressions of several genes encoding these enzymes, therefore guide the metabolic flux towards certain pathways (Ying et al ., 2019). It is an effective methodology using TFs to improve the production of secondary metabolites in plants. Several MYBs have been reported to regulate tanshinones biosynthesis of S. miltiorrhiza. Overexpression of $S m M Y B 36$ promoted tanshinones accumulation by binding to the promoters of $D X R, M C T$ and GGPPS1 (Ding et al ., 2017). Overexpression of $S m M Y B 9 b$ also increases tanshinones concentration by stimulating the MEP pathway (Zhang et al. , 2017). And overexpression of SmbHLH10 enhanced the tanshinones biosynthesis by binding with the G-box of DXS2 and CPS5 in S. miltiorrhiza (Xinget al ., 2018). Moreover, SmWRKY1 could positively regulate tanshinones biosynthesis via target gene $D X R$ in $S$. miltiorrhiza (Cao et al. , 2018). But ethylene response factor (ERF), SmERF115 decreased tanshinones content in S. miltiorrhiza(Sun et al. , 2018). However, the regulatory function of GA signaling key regulator SmGRASs in tanshinones biosynthesis of S. miltiorrhiza remains unknown.

GRAS TF family has been reported playing diverse roles in GA signaling, root development, light signaling and stress responses (Livne et al. , 2015; Xu et al. , 2015; Heck et al. , 2016). GRAS has been found in many plants, such as Arabidopsis , tomato, rice, grapevine, cotton and Danshen (Tian et al. , 2004; Huang et al. , 2015; Grimplet et al. , 2016; Bai et al. , 2017; Zhanget al. , 2018). The GRAS family is divided into 13 distinct subfamilies based on amino acid sequences: DELLA, SCL3, LAS, SCL28, SCL4/7, SCR, SHR, SCL9 (LISCL), HAM, PAT1, OS4, DLT and OS19 (Zhanget al. , 2018). Among them, the SCL3 subfamily has been shown to participate in root cell elongation, GA/DELLA signaling and stress responses (Hakoshima, 2018). Furthermore, some evidence has shown that GRAS proteins participated in the GAdependent regulatory network and root periderm formation. For instance, DELLA protein is the repressor of GA and acted as key regulatory targets in the GA signaling pathway in regulating plant growth (Murase et al. , 2008; Yoshida et al. , 2014). SCL3 has been reported as a repressor of DELLA. It could positively regulate the GA signaling pathway and control GA homeostasis in Arabidopsis root development (Heo et al. , 2011; Zhanget al. , 2011).

GA is an important phytohormone that plays vital roles in many processes of plant growth and metabolism (Brian, 2010; Du et al. , 2015; Davière and Achard, 2016). For instance, GA could regulate the flavonol biosynthesis through DELLA to further promote root growth in Arabidopsis (Tan et al. , 2019). And the GA-mediated control of growth has an interaction with energy metabolism to coordinates cell wall extension, lipid and secondary metabolism in Arabidopsis (Ribeiro et al. , 2012). Moreover, the biosynthesis pathways of GA and tanshinones are different branches of the diterpenoid biosynthesis pathway, since they have a universal precursor GGPP (Xu et al ., 2015). The biosynthesis of GA from GGPP involves many synthase genes, such as CPS5 ,KS , KAO , GA20ox, GA3ox and GA2ox (Maet al. , 2012; Cui et al. , 2015; Su et al. , 2016). Thus, crosstalk may occur between GA and tanshinones biosynthesis inS. miltiorrhiza . In our previous report, GA treatment was able to increase tanshinones accumulation and induce the expressions of $S m G R A S 1^{\sim} 5$ genes in wild-type hairy roots of $S$. miltiorrhiza (Bai et al. , 2018). Therefore, we speculated that SmGRASs might be involved in the regulation of GA to tanshinones biosynthesis in $S$. miltiorrhiza . Although five GRAS family genes have been cloned in $S$. miltiorrhiza, however, we found the $S m G R A S 5$ was the most sensitive gene of them responding to GA treatment (Bai et al. , 2017). But the roles of SmGRAS5 in regulating tanshinones biosynthesis through GA signaling are still unknown.

In this study, we found that overexpressing SmGRAS5 significantly increased the tanshinones' content and 
decreased GA content in S. miltiorrhiza hairy roots. Y1H, Dual-LUC and EMSA assays indicated that SmGRAS5 could directly bind to the promoter of SmKSL1 to induce its expression. And GA treatment improved the tanshinones and GA accumulation in the SmGRAS5 OE lines. Subsequently, transcriptome analysis revealed the potential functions of SmGRAS5 in regulating secondary metabolism. Finally, the molecular mechanism of SmGRAS5 regulated the GA-induced tanshinones biosynthesis was analyzed and discussed. Our findings revealed a link between SmGRAS and secondary metabolism and provided important information on GA-mediated secondary metabolite biosynthesis in S. miltiorrhiza .

\section{MATERIALS AND METHODS}

\section{Plant materials and GA treatment}

Agrobacterium rhizogenes (ATCC15834) was used to infect the terile plantlets to obtain the S. miltiorrhiza hairy roots, as previously reported ( $\mathrm{Ru}$ et al. , 2016). (0.3 $\mathrm{g}$ fresh weight hairy roots were cultured in the liquid 6,7-V medium and sub-cultured every 30 days. The 21-day-old hairy roots were treated with $\mathrm{GA}_{3}(100$ $\mu \mathrm{M}$ final concentration) for $2 \mathrm{~h}, 24 \mathrm{~h}$ or 6 days. Control hairy roots were not treated without $\mathrm{GA}_{3}$. Three independent biological replicates were performed in all treatments.

The phloem tissue, xylem tissue and periderm tissue of the roots were collected from the 2-year-old $S$. miltiorrhiza to analyze the tissue-specific expressions of SmGRAS5 and the tanshinones accumulation.

\section{Bioinformatics analysis of SmGRAS5}

ClustalX program was used to perform multiple sequence alignments of SmGRAS5 (GenBank accession number KY435890) protein sequences and AtGRAS protein sequences (http://www.arabidopsis.org). MEGA6 software was used to construct a phylogenetic tree by the maximum-likelihood method with the bootstrap test ( $\mathrm{n}=500$ replications) (Tamura et al. , 2013).

\section{RNA extraction and qRT-PCR assays}

RNAprep pure plant kit (TIANGEN, China) was used to extracted total RNA and then using the PrimeScript RT reagent kit (TaKaRa, China) to reverse-transcribed RNA to cDNA. SYBR Premix Ex Taq II Kit (Takara, China) was used to performed a real-time PCR system (Bio-Rad CFX96, USA). The SmActin gene was selected as endogenous control (Yanget al. , 2010). $2^{\text {-t }}$ method was used to calculate the relative expression levels. All the qRT-PCR analysis primers are listed in Supplemental Table S1. Three independent biological replicates and three technical replicates were used to obtain the data.

\section{High-performance liquid chromatography (HPLC) analysis of tanshinones and GA $_{3}$ contents}

The contents of tanshinones and $\mathrm{GA}_{3}$ were determined by HPLC, according to the general method in our laboratory that was described previously (Wang et al. , 2008; Liu et al. , 2016). $0.04 \mathrm{~g}$ dried hairy roots power were extracted by $8 \mathrm{~mL}$ of $70 \%$ methanol overnight. After centrifugation, the supernatant was filtered through a $0.2-\mu \mathrm{m}$ filter for HPLC analysis.

\section{Subcellular localization}

The full-length open reading frame (ORF) of SmGRAS5 was fused with GFP in the $p A 7$-GFP vector. Gene gun (Bio-Rad, USA) was used to transform the $p A 7$-SmGRAS5-GFP and control plasmids transiently into onion epidermis. The onion epidermis was stained with 4,6-diamidino-2-phenylindole dihydrochloride (Solarbio, China) for 20 min after 1 day of incubation. And then washed the epidermis twice with PBS buffer (pH 7.2) and observed under a confocal laser scanning microscope (Nikon A1R, Japan).

The $p A 7$ 0390-SmGRAS5-GFP and control plasmids were transformed into Agrobacterium GV3101 . 4week-old $N$. benthamiana leaves were infiltrated with the GV3101 suspension cultures, following the previously described method (Bai et al. , 2018). The protoplasts were prepared after 2 days of co-culture, as previously described (Li, 2011). The subcellular localization analysis primers are listed in Supplemental Table S2. 


\section{Analysis of transcriptional activity}

The $p D E S T$-GBKT7-SmGRAS5 and negative control plasmids were transformed into the yeast AH109. The positive control plasmid was $p G B K T$ 7 $-53+p G A D T 7-T$. First, screened the transformed AH109 on synthetic dropout (SD) medium lacking tryptophan (SD/-Trp) and then on SD medium without tryptophan, histidine and adenine (SD/-Trp/-His/-Ade).

\section{Plasmid construction and genetic transformation}

The ORF of SmGRAS5 was amplified and cloned into the Noc I and Spe I restriction sites of the $p C A M$ BIA1304 in sense and antisense orientations with the CaMV35S promoter. PCR and restriction enzyme digestion were used to confirm the positive clones. And then the plasmids were transformed into ATCC15834 . The combination of cefotaxime (Sigma, USA) and hygromycin B (MP Bio, USA) was used to screen the transformants. PCR identification and the positive transgenic lines screening use four primer pairs, rolB , rolC , hptII , 35S forward primer (35S-F) and GRAS5 reverse primer (GRAS5-R). All the expression vector construction and the PCR identification primers are listed in Supplementary Table S2.

\section{Y1H verification}

The ORF of SmGRAS5 was inserted into the $p G A D T 7$ vector. The 649-bp SmKSL1 and 826-bp SmCPS1 promoter sequences were cloned into the $p B a i t-A b A i$ vector. The primers for vectors ( $p B a i t-A b A i-S m K S L 1$ 649 and $p$ Bait-AbAi-SmCPS1-826) are listed in Table S2. Aureobasidin A suppressed the basal expression of the Y1H-pAbAi-SmKSL1-649 (PYK649) and Y1H-pAbAi-SmCPS1-649 (PYC826) yeast strain (Bai et al. , 2018). pGADT7-SmGRAS5 was verified by interactions withPYK649 and PYC826 yeast strains, which recombined theSmKSL1 and SmCPS1 promoter in SD/-Leu/AbA.

\section{Protein extraction and western blot}

The ORF of SmGRAS5 was cloned into the $p M A L-c 2 X$ vector. The primers for vectors are listed in Table S2. The plasmids were transformed and proteins were expressed inEscherichia coli (Rosetta strain). Protein induction and purification were performed as previously described (Bai et al. , 2018). The MBP (malE) and SmGRAS5-MBP purified proteins were conducted and SDS-PAGE analyses showed major bands with approximate $80.7 \mathrm{kDa}$ molecular mass (Fig. S7). The western blot was performed as previously described (Ruet al. , 2017).

\section{Dual-luciferase assay}

The 649-bp promoter of SmKSL1 was cloned and inserted intopGREEN . The vector $p C A M B I A 1304$ SmGRAS5 was transferred into Agrobacterium strain GV3101. pCAMBIA1304 empty vector was used as a negative control and The 35S promoter-drivenRenilla luciferase as an internal control. The two GV3101 strains were co-infiltrated into tobacco leaves. Infiltrated leaves were incubated in darkness for $8 \mathrm{~h}$ and then in light for 40h. Three biological replicates of each sample were assayed using the Dual-Luciferase Reporter Assay System (Promega, USA).

\section{EMSA analysis}

EMSA kit (Invitrogen, E33075) was used to perform EMSA analysis. The oligonucleotide probes were synthesized (listed in Table S2) and annealed. The protein-free sample was used as blank control. The mass ratios of the probe and protein were 1:5/1:15/1:50 in each reaction mixture $(10 \mu \mathrm{L})$. ChemiDoc XRS+ system (Bio-Rad, USA) was used to observe the gels on a $490 \mathrm{~nm}$ SYBR photographic filter.

\section{Construction of RNA-seq libraries and transcriptome data analysis}

21-day-old transgenic hairy roots (G5O14) and control roots (ATCC) were collected from three biological replicates and used for total RNA extraction. $3 \mu \mathrm{g}$ RNA of each sample was used to construct Illumina sequencing libraries using the Illumina PE150 platform (Novogene, China.). Three biological replicates were used for each sample. 
The reference genome and gene model annotation files were downloaded from genome websites (Xu et al. , 2016). Genes with an adjusted $P$-value $<0.05$ and |fold-change|[?]2 were considered significantly differentially expressed. GO Slim enrichment was evaluated using the GOseq R package (Young et al. , 2010). Significantly enriched KEGG pathways were identified with KOBAS 2.0 (Maoet al. , 2005).

\section{RESULTS}

\section{SmGRAS5 and tanshinones are co-located in the periderm of $S$. miltiorrhiza roots}

To study the functions of SmGRAS5 gene, the coding regions were amplified by using root-derived cDNAs from S. miltiorrhiza .SmGRAS5 contains an open reading frame (ORF) encoding 335 amino acids. The amino acid sequences of SmGRAS5 contained the conserved GRAS domain. The phylogenetic analysis indicated that SmGRAS5 belongs to SCL3 subfamily (Fig. S1).

Since the medicinal part of S. miltiorrhiza is the roots and the tanshinones were mainly accumulated in the roots, the potential functions of SmGRAS5 in tanshinones biosynthesis were detected by analyzing its expression patterns in the periderm, phloem and xylem tissues of $S$. miltiorrhiza roots. The expression levels of SmGRAS5 were the highest in the periderm, suggesting the important roles ofSmGRAS5 in the periderm (Fig. 1A). In addition, the four contents of tanshinones (DT-I, CT, T-I and T-IIA) were also detected in the three tissues of the roots. Tanshinones were mainly concentrated in the periderm (Fig. 1B). The results showed SmGRAS5 to be co-located with tanshinones in the periderm (Fig. 1C). Therefore, we speculated that SmGRAS5 might be involved in the regulation of tanshinones biosynthesis.

\section{Subcellular localization and transcriptional activity analysis of SmGRAS5}

To determine the subcellular localization of SmGRAS5, SmGRAS5 protein was fused with a green fluorescent protein (GFP) label. The protoplasts of tobacco leaves and onion epidermal cells were used to analyze the SmGRA5-GFP signal (Fig. 2). The results showed that the GFP fluorescence of SmGRAS5 was enriched in the nucleus, indicating that SmGRAS5 might function as a TF.

To further identify the characteristics of SmGRAS5, the transcriptional activity of SmGRAS5 was analyzed. The results showed that the yeast withSmGRAS5-pGBKT7 and control constructs were able to grow on the SD/-Trp medium. However, the yeast with SmGRAS5-pGBKTr7 and negative control constructs could not survive on the SD/-Trp-His-Ade medium (Fig. S2). The results demonstrated that SmGRAS5 had no transcriptional activity.

\section{SmGRAS5 promotes tanshinones biosynthesis in $S$. miltiorrhiza}

To further explore the roles of SmGRAS5 in the biosynthesis of tanshinones, the transgenic hairy roots lines were generated through overexpression and antisense expression approaches. The positive transgenic hairy roots were identified by PCR (Fig. S3). Control hairy roots were developed using ATCC15834 without plasmids. Three independent $\mathrm{OE}$ and three AE lines were randomly selected for functional analysis (G5O14, G5O17, G5O20 and G5A11, G5A16, G5A21). Collectively, the gene expression levels of SmGRAS5 were 18to 50 -fold higher in the $\mathrm{OE}$ lines than in the control but decreased by $70 \%-85 \%$ in the AE lines (Fig. 3A).

The phenotypes of control and SmGRAS5 transgenic hairy roots are shown in Figure S4. The SmGRAS5 OE lines showed redder and smaller compared with the control. The HPLC analysis showed that the contents of four tanshinones (DT-I, CT, T-I, T-IIA) were all significantly increased in the SmGRAS5 OE lines (Fig. 3B). Tanshinones' contents were the highest in the G5O14 lines, reaching 5-fold (DT-I), 10.85-fold (CT), 2.94-fold (T-I), and 3.77-fold (T-IIA) of the control lines, respectively. In contrast, the contents of four tanshinones were reduced in the SmGRAS5 AE lines, especially T-I (Fig. 3C). The results showed that SmGRAS5 promoted the accumulation of tanshinones.

To further confirm these results, we then analyzed the expression levels of key genes in the biosynthetic pathways of tanshinones (Fig. 3D). As expected, the expressions of the genes whose promoters contained the GA response motif were consistent with HPLC results. Expressions of most of the key genes in the tanshinones biosynthetic pathway were upregulated in the SmGRAS 5 OE lines. The expressions of downstream genes 
of the tanshinones biosynthetic pathway, such as GGPPS1,CPS1 and KSL1, were indeed significantly upregulated in the SmGRAS 5 OE lines. In contrast, expressions of most of the tanshinones biosynthetic pathway genes were decreased in the AE lines, especially AACT2, HDS , CMK and KSL1 . These data showed that SmGRAS5 could regulate the biosynthesis of tanshinones through regulating the expressions of key biosynthesis genes. Collectively, our findings indicated that SmGRAS5 acted as a positive regulator of tanshinones biosynthesis in S. miltiorrhiza hairy roots.

\section{SmGRAS5 regulates GA-promoted biosynthesis of tanshinones}

Since SmGRAS5 OE lines grow slower than the control, which was similar to the GA-deficient phenotypes (Fig. S4). Consistent with the phenotypes, overexpression of SmGRAS5 indeed decreased the GA content and downregulated the expressions of most GA biosynthetic genes (Fig. 4A,C). To further investigate whether the regulatory function of SmGRAS5 on tanshinones biosynthesis is mediated by GA, we then used exogenous GA to treat the SmGRAS5 OE and control lines. We found that there are some GA-responded motifs (GARE-motif and P-box) in the promoter of SmGRAS5. And we also found GA treatment could induce the expression of SmGRAS5 (Fig. S5). The results showed that the GA content of control and SmGRAS5 OE lines were all significantly increased after GA treated. However, the expressions of the downstream genes of GA biosynthesis were different in the SmGRAS5 OE lines. GA biosynthetic enzymes SmGA20ox2/6 were upregulated, whileSmGA3ox1 was downregulated in the SmGRAS5 OE lines. And the expressions of the GA deactivating enzymes SmGA20x8/9 were different in the SmGRAS5 OE lines. The results after GA treatment indicated that the increased GA content in the SmGRAS5 OE lines maybe not have occurred by promoting GA biosynthesis but could have also been caused by exogenous GA entering the cell. Intriguingly, the four tanshinones total contents of SmGRAS5 OE and control lines were all significantly increased under GA treatments (Fig. 4B). As expected, the expression of most tanshinones biosynthesis genes was quickly induced by GA application in all the lines (Fig. 4C). Among the tanshinones biosynthesis genes, the downstream genes GGPPS1 and KSL1 had the most significantly increased in the SmGRAS5 OE and control lines. Collectively, these results indicated that GA could induceSmGRAS5 response to further promote tanshinone biosynthesis.

\section{Interaction of SmGRAS5 with tanshinones and GA biosynthetic gene}

To further investigate how SmGRAS5 regulates tanshinones biosynthesis, we performed Y1H, Dual-LUC and EMS assays. According to our qRT-PCR results, the expressions of most genes in the tanshinones biosynthetic pathway were upregulated in the SmGRAS5 OE lines. Among them,SmCPS1 and SmKSL1, which are the key downstream genes in tanshinones biosynthesis, their expressions were remarkably upregulated. The Y1H Gold reporter strains that have the SmKSL1 promoter transformed with SmGRAS5 prey plasmid and positive control could grow normally on SD/-Leu (700 ng/ml AbA) (Fig. 5A). However, the Y1H Gold reporter strains that have the SmCPS1 promoter transformed with SmGRAS5 prey plasmid and negative control could not grow on SD/-Leu (700 ng/ml AbA) (Fig. S6). These results provided in vivo evidence that SmGRAS5 participates in tanshinones biosynthesis by directly binding to the SmKSL1 promoter. We further confirmed this by dual-luciferase report system. The Dual-LUC assay showed that SmGRAS5 could directly activate the SmKSL1 promoter (Fig. 5B).

Our promoter analysis indicated that the promoter sequence of $S m K S L 1$ contains a GARE motif at 774 bp upstream of the ATG codon (Fig. 5C). To further confirm binding between SmGRAS5 and the fragment containing the SmGRAS5 recognition element in vitro, purified SmGRAS5-MBP fusion proteins were combined with the fragment containing the GARE motif, and they were analyzed by EMSA. Subsequently, specific DNA-SmGRAS5 protein complexes were strongly detected (Fig. 5C). These results confirmed that SmGRAS5 regulated tanshinones biosynthesis by directly binding to the GARE motif of the SmKSL1 promoter. All these data together indicated that SmGRAS5 could directly bind to the GRAE in the promoter of SmKSL1 to induce its expression.

Furthermore, in order to further study whether SmGRAS5 directly regulates the expressions of GA biosynthesis genes. We analyzed some promoters of GA biosynthesis genes that were significantly downregulated 
by SmGRAS5. We designed GA response element probes for some GA biosynthesis genes and conducted EMSA experiments (Fig. S8). Unfortunately, none of the promoters of these GA biosynthesis genes interacted with SmGRAS5. We speculated that SmGRAS5 might inhibit the expressions of GA biosynthesis genes by interacting with other negative regulatory factors or inhibit GA biosynthesis by other pathways.

\section{Transcriptome analysis of SmGRAS5-regulated genes}

To gain a comprehensive overview of the changes regulated by SmGRAS5 at the transcript level, we performed a transcriptome analysis of G5O14 and ATCC hairy roots. A total of 3910 differentially expressed genes (DEGs) were designated as SmGRAS5-regulated genes (Fig. 6A). A functional gene ontology (GO) analysis indicated that the DEGs regulated by SmGRAS5 were mostly enriched in biological process, metabolic process and catalytic activity terms (Fig. 6B). The Kyoto Encyclopedia of Genes and Genomes (KEGG) analysis showed that hundreds of genes for metabolic pathways and the biosynthesis of secondary metabolites were enriched. Moreover, there were many DEGs enriched in stilbenoid, diarylheptanoid and gingerol biosynthesis, phenylpropanoid biosynthesis, limonene and pinene degradation, flavonoid biosynthesis, diterpenoid biosynthesis and plant hormone signal transduction (Fig. 6C). Since many genes involved in secondary metabolites biosynthesis were identified as SmGRAS5 regulated genes in our transcriptomic results. We further analyzed the secondary metabolism pathway using Mapman software (Fig. 6D). We could see that most of the DEGs in MVA and non MVA pathways were upregulated. And there were more upregulated DEGs in the terpenoids, alkaloids-like, carotenoids, flavonoids, anthocyanins and glucosinolates pathways. However, most DEGs in wax and chalcones pathways were downregulated.

Furthermore, we further analyzed transcriptome data to determine the regulatory roles of SmGRAS5 in tanshinones, GA biosynthetic and GA signaling pathways. As shown in Figure 7, the expressions of most tanshinones biosynthetic pathway genes were upregulated while the expressions of most GA biosynthetic and signaling pathway genes were downregulated in the SmGRAS5 OE lines. The qRT-PCR analysis verified the transcriptomic data (Fig. S9). These results were consistent with our above data and supported the function of SmGRAS5 in promoting tanshinones biosynthesis but inhibiting the GA biosynthesis and GA signaling pathway. Taken together, our results suggested that SmGRAS5 might reduce GA biosynthesis by promoting the universal precursor GGPP to synthesize more tanshinones and further to inhibit downstream GA signaling pathway.

\section{DISCUSSION}

\section{GA signaling involved in the biosynthesis of tanshinones and SAs}

GA is a diterpene phytohormone that modulates growth and development throughout the whole life cycle of the plant (Sun, 2011). A study has shown that GA could promote the accumulation of tanshinones in the wild-type hairy roots of S. miltiorrhiza (Bai et al. , 2017), but its regulatory mechanisms are poorly understood. We found the SmGRAS5 was the most sensitive genes to GA response of the five SmGRASs genes in our previous study (Bai et al. , 2017). We speculated that it might play a major role in SmGRASs in the GA signaling. As a key regulator of GA signaling, GRAS has been classified into 13 different subgroups in Arabidopsis (Zhang et al ., 2018). Our phylogenetic analysis showed SmGRAS5 belonged to SCL3 subfamily, which is involved in the regulation of Arabidopsis root cell elongation, GA homeostasis and signaling (Heo et al. , 2011). AtSCL3 inhibited GA biosynthesis and antagonized with DELLA in maintaining GA homeostasis by feedback regulating upstream GA biosynthesis genes, as well as in modulating downstream GA responses by direct protein-protein interaction (Zhang et al. , 2011). DELLA protein interacted with AtMYB12 activating expression of flavonol biosynthetic genes (Tan et al. , 2019). The tissue-specific expression of GRAS genes often indicates their functional roles in development. It has been reported that diterpenoid tanshinones not only accumulated but also biosynthesized in the roots periderm ofS. miltiorrhiza (Xu et al. , 2015). We found that SmGRAS5 highly expressed in the periderm and co-localized with tanshinones, which indicated that SmGRAS5 might be involved in the regulation of tanshinones biosynthesis. Overexpression of SmGRAS5significantly improved tanshinones accumulation through upregulating the expressions of biosynthetic genes, while in the SmGRAS5 AE lines the tanshinones accumulation was decreased. Notably, 
the promotion of SmGRAS5 to the tanshinones biosynthesis is the most significant in all of the SmGRASs, which we are currently studying. So we speculated that it might play a vital role in the regulation of tanshinones biosynthesis. Considering the correlation between tanshinones and GA biosynthetic pathways, we found that the expressions of the most of GA biosynthetic pathway genes were downregulated, which resulted in the decrease of GA content in the SmGRAS5 OE lines. Similarly, overexpressing HaGRASL also reduced the metabolic flow of GAs in Arabidopsis (Fambrini et al. , 2015). SilencingSlGRAS26 inhibited the GA biosynthetic pathway, promoted the GA inactivation pathway, and resulted in GA deficiency in tomato (Zhouet al. , 2018). SlGRAS24 could downregulate GA biosynthetic genes, disrupt GA homeostasis and participate in a series of developmental processes through modulating GA and auxin signaling (Huanget al. , 2017). We speculated that SmGRAS5 might regulate the metabolic flow of diterpenoid GA and tanshinones biosynthesis. In addition, the GA application could promote the tanshinones biosynthesis in SmGRAS5 OE lines as well as control lines. Therefore, these results implied that SmGRAS5 could be induced by GA and might catalyze the precursor GGPP to synthesize more tanshinones. Moreover, transcriptome data also revealed that SmGRAS5 could regulate many secondary metabolites biosynthesis. It could inhibit the GA biosynthetic and signaling pathway at the same time, and induce tanshinones biosynthetic pathway through regulating biosynthetic genes.

\section{SmGRAS5 directly regulated tanshinones biosynthetic gene but not GA}

TFs usually regulate the transcription of target genes by directly binding to the elements in the promoter. Studies have shown that GRAS could interact with the promoter of downstream genes and regulate their expression (Smit et al. , 2005; Hirsch et al. , 2009). For instance, OsGRAS23 could bind to the potential target genes' promoters to further positively modulating rice drought tolerance (Xu et al. , 2015). AtSCL14 was shown to be essential for activating the stress-inducible promoters (Fode et al. , 2008). Moreover, SlGRAS2 also regulated the fruit development downstream genes (Li et al. , 2018). Therefore, we speculated that SmGRAS5 might directly regulate the expressions of the tanshinones and GA biosynthetic pathway genes. Previous work has indicated that tanshinones biosynthesis is initiated by cyclization of general diterpenoid precursor GGPP to copalyl diphosphate (CPP) by SmCPS1 and subsequent further cyclization to the abietane miltiradiene by SmKSL1 (Cui et al. , 2015).SmCPS1 and KSL1, as the downstream key enzyme genes in tanshinones biosynthetic pathway, were the most highly expressed in the periderm and played a vital role in tanshinones biosynthesis (Xuet al. , 2015). Considering the significant response of SmCPS1 and SmKSL1 to SmGRAS5 and GA, Y1H, Dual-LUC and EMSA assays were performed and demonstrated that SmGRAS5 recognized the GARE motif of the SmKSL1 promoter, leading to the activation of expression. However, we verified several GA biosynthetic genes and found that none of their promoters interacted with SmGRAS5. These results showed that SmGRAS5 could only directly regulate tanshinones biosynthesis by interacting with SmKSL1. However, SmKSL1 was likely not the only target gene for SmGRAS5 regulation. GRAS could also interact with other TFs to mediate the regulation of the transcription activity of other target genes. For instance, GA inhibited flavonol biosynthesis via DELLA protein, which could interact with SG7 MYBs, to regulate the transcriptional levels of the biosynthesis pathway key genes (Tan et al. , 2019). In addition, the specific regulatory mechanism of SmGRAS5 on GA biosynthesis genes is also unclear. SmGRAS was likely to co-regulate GA biosynthesis by interacting with other negative regulatory factors and forming complexes. Therefore, identifying new interactive TFs or downstream targets of SmGRAS5 may provide further insight into the molecular mechanism of SmGRAS5-mediated regulation of secondary metabolite biosynthesis.

Finally, a potential mechanism for the SmGRAS5-mediated GA-promoted tanshinones biosynthesis was proposed (Fig. 8). GA could induceSmGRAS5 response and promote the biosynthesis of tanshinones through directly activating the expression of SmKSL1. SmGRAS5 could inhibit the GA biosynthetic and signaling pathway at the same time and might promote metabolic flow to catalyze the precursor GGPP to synthesize more tanshinones.

\section{The importance of SmGRAS5 for metabolic engineering}

The biosynthesis of secondary metabolites usually involves multiple enzymes. We can promote the accumula- 
tion of secondary metabolites by regulating the expressions of these enzymes. Overexpression of artemisinin biosynthesis gene FPS , CYP $71 A V 1$, DXR significantly increased the artemisinin content (Chen et al. , 2000; Shenet al. , 2012; Xiang et al. , 2012). Overexpressing the key enzyme gene HMGR in the MVA pathway increased the content of ajmalicine and catharanthine in Catharanthus roseus hairy roots (AyoraTalavera et al. , 2002). However, in most biosynthetic pathways, the rate-limiting step is not limited to one or two enzymes. TFs often target the key steps in biosynthetic pathways. Many TFs, such as MYB, AP2/ERF, bHLH, and WRKY, have been reported to have global regulatory functions in pharmaceutical terpenoid biosynthesis (Xieet al. , 2012; Liu et al. , 2015; Lu et al. , 2016). CrWRKY1, TcWRKY1 and AaWRKY1 can significantly improve the content of serpentine, taxol and artemisinin biosynthesis in Catharanthus roseus, Taxus chinensis and Artemisia annua, respectively (Suttipanta et al ., 2011; Li et al ., 2012; Ma et al. , 2009). Overexpression of AaORA in Artemisia annuasignificantly increased the expressions of several biosynthetic genes including $A D S, C Y P 71 A V 1$, and DBR2, and led to a significant increase in artemisinin production (Lu et al. , 2013). SmGRAS5 as a positive regulator mediated GA-promoted diterpenoid compound tanshinines biosynthesis by regulating the expression of SmKSL1 and induce the anthocyanin, diterpenoid compound, artemisinin, and triterpene saponins, cannabidiolic acid biosynthetic genes in transcriptome data (Solfanelli et al. , 2006; Han et al. , 2013; Weiblen et al. , 2015; Guoet al. , 2016; Xiang et al. , 2012). Therefore, make full use of TFs as a general tool to control metabolic pathways, is vital for successful metabolic engineering.

\section{ACKNOWLEDGMENTS}

This work was supported by the National Natural Science Foundation of China (No.81373908, 81973415 and 81673536) and the Ministry of Science and Technology of the Twelfth Five-Year National Science and Technology Pillar Program (No.2015BAC01B03).

\section{CONFLICT OF INTEREST}

The authors have declared no conflict of interest.

\section{AUTHOR CONTRIBUTIONS}

W.L., Z.B. and Z.L. conceived and designed the experiment; W.L., Z.B., T.P., R.M. and R.H. performed the experiments; W.L. analyzed data; W.L. and D.Y.wrote the article.

\section{REFERENCES}

Brian P.W. (2010) Effects of gibberellins on plant growth and development. Biological Reviews 34, 37-77. Cao W.Z., Wang Y., Shi M., Hao X.L., Zhao W.W., Wang Y., Ren J. \& Kai G.Y. (2018) Transcription Factor SmWRKY1 positively promotes the biosynthesis of tanshinones in Salvia miltiorrhiza.Frontiers in Plant Science 9, 554. Chen D.H., Ye H.C. \& Li G.F. (2000) Expression of a chimeric farnesyl diphosphate synthase gene in Artemisia annua L. transgenicplants via Agrobacterium tumefaciens-mediated transformation. Plant Science 155, 179-185. Cui G.H., Duan L.X., Jin B.L., Qian J., Xue Z.Y., Shen G., Syder J.H., Song J.Y., Chen S.L., Huang L.Q., Qi X.Q. \& Peter R.J. (2015) Functional divergence of diterpene syntheses in the medicinal plantSalvia miltiorrhiza. Plant Physiology 169, 1607-1618. Davière J-M. \& Achard P. (2016) A pivotal role of DELLAs in regulating multiple hormone signals. Molecular Plant 9, 10-20. Ding K., Pei T.L., Bai Z.Q., Jia Y.Y., Ma P.D. \& Liang Z.S. (2017) SmMYB36, a novel R2R3-MYB transcription factor, enhances tanshinone accumulation and decreases phenolic acid content in Salvia miltiorrhiza hairy roots.Scientific Reports 7, 5104. Dong Y.Z., Morris-natschke S.L., Lee K-H. (2011) Biosynthesis, total syntheses, and antitumor activity of tanshinones and their analogs as potential therapeutic agents.Natural Product Reports 28, 529-542. Du Q., Li C.L., Li D.Q. \& Lu S.F. (2015) Genome-wide analysis, molecular cloning and expression profiling reveal tissue-specifically expressed, feedback-regulated, stress-responsive and alternatively spliced novel genes involved in gibberellin metabolism in Salvia miltiorrhiza. BMC Genomics 16, 1087. Fambrini M., Mariotti L., Parlanti S., Salvini M. \& Pugliesi C. (2015) A GRAS-like gene of sunflower 
(Helianthus annuus L.) alters the gibberellin content and axillary meristem outgrowth in transgenic Arabidopsis plants. Plant Biology 17, 1123-1134. Fode B., Siemsen T., Corinna T., Weigel R. \& Gatz C. (2008) The Arabidopsis GRAS protein SCL14 interacts with class II TGA transcription factors and is essential for the activation of stress-inducible promoters. Plant Cell20, 3122-3135. Fu R., Martin C. \& Zhang Y.(2018) Next-generation plant metabolic engineering, inspired by an ancient Chinese irrigation system. Molecular Plant11, 47-57. Grimplet J., Agudelo-romero P., Teixeira R.T., Martinez-zapater J.M. \& Fortes A.M. (2016) Structural and functional analysis of the GRAS gene family in grapevine indicates a role of GRAS proteins in the control of development and stress responses. Frontiers in Plant Scirnce 7, 353-374. Guo J., Ma X.H., Cai Y., Ma Y., Zhan Z.L., Zhou Y.J., Liu W.J, Guan M.X., Yang J., Cui G.H., Kang L.P., Yang L., Shen Y., Tang J.F., Lin H.X., Ma X.J., Jin B.L., Liu Z.M., Peter R.J., Zhao Z.K. \& Huang L.Q. (2016) Cytochrome P450 promiscuity leads to a bifurcating biosynthetic pathway for tanshinones. New Phytologist 210, 525-534. Hakoshima T. (2018) Structural basis of the specific interactions of GRAS family proteins.FEBS Letters 592, 489-501. Han J-Y., Kim M-J., Ban Y-W., Hwang H-S. \& Choi Y-E. (2013) The involvement of $\beta$-amyrin 28-oxidase (CYP716A52v2) in oleanane-type ginsenoside biosynthesis in Panax ginseng.Plant $\mathcal{E}$ Cell Physiology 54, 2034-2046. Heck C., Kuhn H., Heidt S., Walter S., Rieger N. \& Requena N. (2016) Symbiotic fungi control plant root cortex development through the novel GRAS transcription factor MIG1.Current Biology 26, 2770-2778. Heo J-O., Chang K.S., Kim I.A., Lee M-H., Lee S.A., Song S-K., Lee M.M. \& Lim J. (2011) Funneling of gibberellin signaling by the GRAS transcription regulator SCARECROW-LIKE 3 in the Arabidopsis root. Proc Natl Acad Sci USA108, 2167-2171. Hirsch S., Kim J., Muñoz A., Heckmann A.B., Downie J.A. \& Oldroyd G.E. (2009) GRAS proteins form a DNA binding complex to induce gene expression during nodulation signaling inMedicago truncatula. Plant Cell 21, 545-557. Huang W., Xian Z.Q., Kang X., Tang N. \& Li Z.G. (2015) Genome-wide identification, phylogeny and expression analysis of GRAS gene family in tomato.BMC Plant Biology 15, 209-226. Huang W., Peng S.Y., Xian Z.Q., Lin D.B., Hu G.J., Yang L., Ren M.Z. \& Li Z.G. (2017) Overexpression of a tomato miR171 target gene SlGRAS24 impacts multiple agronomical traits via regulating gibberellin and auxin homeostasis.Plant Biotechnology Journal 15, 472-488. Kai G.Y., Xu H., Zhou C.C., Liao P. \& Zhang L. (2011) Metabolic engineering tanshinone biosynthetic pathway in Salvia miltiorrhiza hairy root cultures. Metabolic Engineering 13, 319-327. Li M., Wang X., Li C.X., Li H.X., Zhang J.H. \& Ye Z.B. (2018) Silencing GRAS2 reduces fruit weight in tomato. Journal of Integrative Plant Biology 60, 498-513. Li S., Zhang P., Zhang M., Fu C. \& Yu L. (2012) Functional analysis of a WRKY transcription factor involved in transcriptional activation of the DBAT gene in taxus chinensis. Plant Biology 15, 19-26. Li X. (2011) A transient expression assay using Arabidopsis mesophyll protoplasts.Bio-Protocol1, e70. Liu H.M., Ma S.L., Xia H.R., Lou H.X., Zhu F.L. \& Sun L.R. (2018) Anti-inflammatory activities and potential mechanisms of phenolic acids isolated from Salvia miltiorrhiza f. alba roots in THP-1 macrophages. Journal of Ethnopharmacology 222, 201-207. Liu J.Y., Osbourn A., Ma P.D. (2015) MYB transcription factors as regulators of phenylpropanoid metabolism in plants. Molecular Plant 8, 689-708. Liu L., Yang D.F., Liang T.Y., Zhang H.H., He Z.G. \& Liang Z.S. (2016) Phosphate starvation promoted the accumulation of phenolic acids by inducing the key enzyme genes inSalvia miltiorrhiza hairy roots.Plant Cell Reports 35, 1933-1942. Livne S., Lor V.S., Nir I., Eliaz N., Aharoni A., Olszewski N.E., Eshed Y. \& Weiss D. (2015) Uncovering DELLA-independent gibberellin responses by characterizing new Tomatoprocera mutants. Plant Cell 27, 1579-1594. Lu X., Tang K.X. \& Li P. (2016) Plant metabolic engineering strategies for the production of pharmaceutical terpenoids.Frontiers in Plant Science 7, 1647. Lu X., Zhang L., Zhang F.Y., Jiang W.M., Shen Q., Zhang L.D., Lv Z.Y., Wang G.F. \& Tang K.X. (2013) AaORA, a trichome-specific AP2/ERF transcription factor of Artemisia annua, is a positive regulator in the artemisinin biosynthetic pathway and in disease resistance toBotrytis cinerea. New Phytologist 198, 1191-1202. Ma DM, Pu GB, Lei CY, Ma L.Q., Wang H.H., Guo Y.W., Chen J.L., Du Z.G., Wang H., Li G.F., Ye H.C. \& Liu B.Y. (2009) Isolation and characterization 
of AaWRKY1, anArtemisia annua transcription factor that regulates the amorpha-4,11-diene synthase gene, a key gene of artemisinin biosynthesis. Plant \& Cell Physiology 50, 2146-2161. Ma X.H., Ma Y., Tang J.F., He Y.L., Liu Y.C., Ma X.J., Shen Y., Cui G.H., Lin H.X., Rong Q.X., Guo J. \& Huang L.Q. (2015) The biosynthetic pathways of tanshinones and phenolic acids in Salvia miltiorrhiza. Molecules20, 16235. Ma Y., Ma X.H., Meng F.Y., Zhan Z.L., Guo J. \& Huang L.Q. (2016) RNA interference targeting CYP76AH1 in hairy roots of Salvia miltiorrhi$z a$ reveals its key role in the biosynthetic pathway of tanshinones.Biochemical and Biophysical Research Communications 477, 155-160. Ma Y.M., Yuan L.C., Wu B., Li X.E., Chen S.L. \& Lu S.F. (2012) Genome-wide identification and characterization of novel genes involved in terpenoid biosynthesis inSalvia miltiorrhiza.Journal of Experimental Botany 63, 2809-2823. Mao X.Z., Cai T., Olyarchuk J.G. \& Wei L.P. (2005) Automated genome annotation and pathway identification using the KEGG Orthology (KO) as a controlled vocabulary.Bioinformatics 21, 3787-3793. Murase K., Hirano Y., Sun T.P. \& Hakoshima T. (2008) Gibberellin-induced DELLA recognition by the gibberellin receptor GID1. Nature 456, 459-463. Pei T.L., Ma P.D., Ding K., Liu S.J., Jia Y.Y., Ru M., Dong J.E. \& Liang Z.S. (2018) SmJAZ8 acts as a core repressor regulating JA-induced biosynthesis of salvianolic acids and tanshinones in Salvia miltiorrhiza hairy roots. Journal of Experimental Botany 69, 1663-1678. Petersen M. (2013) Rosmarinic acid: new aspects. Phytochemistry Reviews 12, 207-227. Ribeiro D.M., Araújo W.L., Fernie A.R., Schippers JHM. \& Mueller-Roeber B. (2012) Translatome and metabolome effects triggered by gibberellins during rosette growth inArabidopsis. Journal of Experimental Botany 63, 2769-2786. Ru M., An Y.Y., Wang K.R., Peng L., Li B., Bai Z.Q., Wang B.Q. \& Liang Z.S. (2016) Prunella vulgaris L. hairy roots: Culture, growth, and elicitation by ethephon and salicylic acid. Engineering in Life Sciences 16, 494-502. Ru M., Wang K.R., Bai Z.Q., Peng L., He S.X., Wang Y. \& Liang Z.S. (2017) A tyrosine aminotransferase involved in rosmarinic acid biosynthesis in Prunella vulgaris L.Scientific Reports 7, 4892. Shen Q., Chen Y.F., Wang T., Wu S.Y., Lu X., Zhang L., Zhang F.Y., Jiang W.M., Wang K.X. \& Tang G.F. (2012) Overexpression of the cytochrome P450 monooxygenase (CYP71AV1) and cytochrome P450 reductase (CPR) genes increased artemisinin content in Artemisia annua (Asteraceae).Genetics and Molecular Research 11, 3298-3309. Shi M., Luo X.Q., Ju G.H., Li L.L., Huang S.X., Zhang T., Wang H.Z. \& Kai G.Y. (2016) Enhanced diterpene tanshinone accumulation and bioactivity of transgenic Salvia miltiorrhiza hairy roots by pathway engineering. Journal of Agricultural and Food Chemistry 64, 2523-2530. Smit P., Raedts J., Portyanko V., Debelle F., Gough C., Bisseling T. \& Geurts R. (2005) NSP1 of the GRAS protein family is essential for Rhizobial Nod factor-induced transcription. Science 308, 17891791. Solfanelli C., Poggi A., Loreti E., Alpi A. \& Perata P. (2006) Sucrose-specific induction of the anthocyanin biosynthetic pathway in Arabidopsis. Plant Physiology 140,637-646. Song Z. \& Li X. (2015) Expression profiles of rosmarinic acid biosynthesis genes in two Salvia miltiorrhiza lines with differing water-soluble phenolic contents. Industrial Crops and Products 71, 24-30. Su P., Tong Y.R., Cheng Q.Q., Hu Y.T., Zhang M., Yang J., Teng Z.Q., Gao W. \& Huang L.Q. (2016) Functional characterization of ent-copalyl diphosphate synthase, kaurene synthase and kaurene oxidase in the Salvia miltiorrhizagibberellin biosynthetic pathway. Scientific Reports 6, 23057. Sun M.H., Shi M., Wang Y., Huang Q., Yuan T.P., Wang Q., Wang C., Zhou W. \& Kai G.Y. (2018) The AP2/ERF transcription factor SmERF115 positively regulates the biosynthesis of phenolic acids in Salvia miltiorrhiza. Journal of Experimental Botany 70, 243-254. Sun T.P. (2011) The molecular mechanism and evolution of the review GA-GID1-DELLA signaling module in plants. Current Biology21, 338-345. Suttipanta N., Pattanaik S., Kulshrestha M., Patra B., Singh S.K. \& Yuan L. (2011) The transcription factor CrWRKY1 positively regulates the terpenoid indole alkaloid biosynthesis in Catharanthus roseus.Plant Physiology 157, 2081-2093. Tamura K., Stecher G., Peterson D., Filipski A. \& Kumar S.(2013) MEGA6: molecular evolutionary genetics analysis version 6.0. Molecular Biology and Evolution 30, 2725-2729. Tan H.J., Man C., Xie Y., Yan J.J., Chu J.F. \& Huang J.R. (2019) A crucial role of GA-regulated flavonol biosynthesis in root growth of Arabidopsis. Molecular Plant 12, 521-537. The State Pharmacopoeia 
Commission of China. (2015) Pharmacopoeia of the People's Republic of China, Part I. pp. 7677. Chemical Industry Press, Beijing. Tian C.G., Wan P., Sun S.H., Li J.Y. \& Chen M.S. (2004) Genome-wide analysis of the GRAS gene family in Rice and Arabidopsis.Plant Molecular Biology 54, 519-532. Wang J., Li M., Zhang Y.H. \& Yang S.C. (2008) HPLC analysis of ABA and $\mathrm{GA}_{3}$ in reproductive organs of Bruguiera gymnorrhiza.Journal of Xiamen University 47, 752-756. Wang X.H., Morris-Natschke S.L. \& Lee K-H. (2007) New developments in the chemistry and biology of the bioactive constituents of Tanshen. Medicinal Research Reviews 27, 133-148. Weiblen G.D., Wenger J.P., Craft K.J., Elsohly M.A., Mehmedic Z., Treiber E.L. \& Marks M.D. (2015) Gene duplication and divergence affecting drug content in Cannabis sativa. New Phytologist 208, 1241-1250. Xiang L., Zeng L.J., Yuan Y., Chen M., Wang F., Liu X.Q., Lan X.Z. \& Liao Z.H. (2012) Enhancement of artemisinin biosynthesis by overexpressing DXR, CYP71AV1 and CPR in the plants of Artemisia annua L. Journal of Plant Molecular Biology $\&$ Omics 5, 503-507. Xie X.B., Li S., Zhang R.F., Zhao J., Chen Y.C., Zhao Q., Yao Y.X., You C.X., Zhang X.S. \& Hao Y.J. (2012) The bHLH transcription factor MdbHLH3 promotes anthocyanin accumulation and fruit colouration in response to low temperature in apples. Plant Cell and Environment35, 1884-1897. Xing B.C., Liang L.J., Liu L., Hou Z.N., Yang D.F., Yan K.J., Zhang X.M. \& Liang Z.S. (2018) Overexpression of SmbHLH148 induced biosynthesis of tanshinones as well as phenolic acids in Salvia miltiorrhiza hairy roots.Plant Cell Reports 37, 1681-1692. Xu H.B., Song J.Y., Luo H.M., Zhang Y.J., Li Q.S., Zhu Y.J., Xu J., Li Y. Song C., Wang B., Sun W., Shen G.A., Zhang X., Qian J., Ji A.J., Xu Z.C., Luo X., He L., Li C.Y., Sun C., Yan H.X., Cui G.H., Li X.W., Li J.H., Liu J.Y., Wang Y.T., Hayward A., Nelson D., Ning Z.M., Peter R.J., Qi X.Q. \& Chen S.L. (2016) Analysis of the genome sequence of the medicinal plant Salvia miltiorrhiza. Molecular Plant9, 949-952. Xu K., Chen S.J., Li T.F., Ma X.S., Liang X.H., Ding X.F., Liu H.Y. \& Luo L.J. (2015) OsGRAS23, a rice GRAS transcription factor gene, is involved in drought stress response through regulating expression of stress-responsive genes.BMC Plant Biology 15, 141. Xu Z.C., Peters R.J., Weirather J., Luo H.M., Liao B.S., Zhang X., Zhu Y.J., Ji A.J., Zhang B., Hu S.N., Au K.F., Song J.Y. \& Chen S.L. (2015) Full-length transcriptome sequences and splice variants obtained by a combination of sequencing platforms applied to different root tissues of Salvia miltiorrhiza and tanshinone biosynthesis. Plant Journal 82, 951-961. Yang Y.F., Hou S., Cui G.H., Chen S.L., Wei J.H. \& Huang L.Q. (2010) Characterization of reference genes for quantitative real-time PCR analysis in various tissues ofSalvia miltiorrhiza.Molecular Biology Reports 37, 507-513. Yoshida H., Hirano K., Sato T., Mitsuda N., Nomoto M., Maeo K., Koketsu E., Mitani R., Kawamura M. \& Ishiguro S. (2014) DELLA protein functions as a transcriptional activator through the DNA binding of the indeterminate domain family proteins. Proc Natl Acad Sci USA 111, 7861-7866. Young M.D., Wakefield M.J., Smyth G.K. \& Oshlack A. (2010) Gene ontology analysis for RNA-seq: accounting for selection bias.Genome Biology 11, R14. Ying S.Y., Su M., Wu Y., Zhou L., Fu R., Li Y., Guo H., Luo J., Wang S.C. \& Zhang Y. (2017) Trichome regulator SIMX1 directly manipulates primary metabolism in tomato fruit. Plant Biotechnology JournalZhang B., Liu J., Yang Z.E., Chen E.Y., Zhang C.J., Zhang X.Y. \& Li F.G. (2018) Genome-wide analysis of GRAS transcription factor gene family in Gossypium hirsutum L..BMC Genomics 19, 348. Zhang J.X., Zhou L.B., Zheng X.Y., Zhang J.J., Yang L., Tan R.H. \& Zhao S.J. (2017) Overexpression of SmMYBgb enhances tanshinone concentration in Salvia miltiorrhiza hairy roots.Plant Cell Reports36, 1297-1309. Zhang L.C., Zhu M.K., Ren L.J., Li A.Z., Chen G.P. \& Hu Z.L. (2018) The SlFSR gene controls fruit shelf-life in tomato. Journal of Experimental Botany 69, 2897-2909. Zhang Z.L., Ogawa M., Fleet C.M., Zentella R., Hu J.H., Heo J-O., Lim J., Kamiya Y., Yamaguchi S. \& Sun T.P. (2011) SCARECROW-LIKE 3 promotes gibberellin signaling by antagonizing master growth repressor DELLA in Arabidopsis.Proc Natl Acad Sci USA 108, 2160-2165. Zhou S.G., Hu Z.L., Li F.F., Yu X.H., Naeem M., Zhang Y.J. \& Chen G.P. (2018) Manipulation of plant architecture and flowering time by down-regulation of the GRAS transcription factor SlGRAS26 inSolanum lycopersicum.Plant Science 271, 81-93. Zhou W., Huang Q., Wu X., Zhou Z.W., Ding M.Q., Shi M., Huang F.F., Li S., Wang Y. \& Kai 
G.Y. (2017) Comprehensive transcriptome profiling of Salvia miltiorrhiza for discovery of genes associated with the biosynthesis of tanshinones and phenolic acids. Scientific Reports 7, 10554.

\section{Figure legends}

Figure 1. Tissue-specific expression of SmGRAS5 and determination of tanshinones. (A) Tissue-specific expression of SmGRAS5 in xylem, phloem and periderm tissues of S. miltiorrhiza roots. The expression levels were normalized to values in the xylem. (B) Contents of tanshinones in different tissues of the roots. (C) A Sectional view of the SmGRAS5 and tanshinones distribution in the root tissues. Standard errors were calculated from three sets of biological replicates. Significant differences using one-way ANOVA and S-N-K comparison tests, $P<0.05$.

Figure 2. Subcellular localization of SmGRAS5 in protoplasts of tobacco leaves (A) and onion epidermal cells (B). Upper images represent the GFP control, while lower images represent the SmGRAS5-GFP fusion proteins. GFP: green fluorescence; DAPI: fluorescence of DAPI nuclear dye; Cholorophyll: chloroplast autofluorescence; Bright field: field observations; Merged: merge of bright field and relevant fluorescence.

Figure 3. SmGRAS5 regulates the biosynthesis of tanshinones in transgenic hairy roots. (A) Relative quantitative analysis of $S m G R A S 5$ expression in the transgenic lines and controls. (B) Analysis of tanshinones production from SmGRAS5 OE hairy root lines. (C) Analysis of tanshinones production from SmGRAS5 AE hairy root lines. (D) Relative expression levels of the genes involved in tanshinones biosyntheses in the SmGRAS5 transgenic lines. Standard errors were calculated from three sets of biological replicates. Significant differences using Student's t-test, ${ }^{*} 0.01<P<0.05,{ }^{* *} P<0.01$.

Figure 4. GA affects the tanshinones and GA contents in SmGRAS5OE hairy roots. (A) Analysis of GA 3 production fromSmGRAS5 OE lines and controls with or without $100 \mu \mathrm{M}$ GA treatment for $6 \mathrm{~d}$. (B) Analysis of tanshinones production from SmGRAS5 OE lines and controls with or without $100 \mu \mathrm{M}$ GA treatment for $6 \mathrm{~d}$. (C) Relative expression levels of genes involved in tanshinones and GA biosyntheses in the SmGRAS5 OE lines with $100 \mu \mathrm{M}$ GA treatment for $2 / 24 \mathrm{~h}$. Standard errors were calculated from three sets of biological replicates. Significant differences using one-way ANOVA and S-N-K comparison tests, $P$ $<0.05$.

Figure 5. SmGRAS5 binds to the GARE motif of the SmKSL1 promoter and activates its expression. (A) Y1H assay shows the interaction between SmGRAS5 and the SmKSL1 promoter. SmKSL1 promoter+pGADT7 as the negative control and SmKSL1 promoter+pGADT7-SmERF6 as positive control. (B) Dual-LUC assay shows the effects of SmGRAS5 on SmKSL1 promoter activation. (C) EMSA analysis of SmGRAS5 binding to the GARE-motif of the SmKSL1 promoter. Schematic diagram showing the GARE motif in the SmKSL1 promoter. Standard errors were calculated from three sets of biological replicates. Significant differences using Student's t-test, ${ }^{*} 0.01<P<0.05$.

Figure 6. Differentially expressed genes (DEGs) regulated by SmGRAS5 from the RNA-seq data. (A) Volcano plot of the DEGs regulated by SmGRAS5 (G5O14 vs ATCC). (B) GO term classifications of the 3910 DEGs from G5O14 vs ATCC. (C) KEGG classification of the 3910 DEGs from G5O14 vs ATCC. (D) Expression levels of representative DEGs of secondary metabolism. The differentially expressed genes demonstrated significantly differential expression (FDR-adjusted $P$-value $<0.05$, |fold-change| [?] 2). The average FPKM ( $\log 10$ scale) of each gene is shown in the heat map.

Figure 7. The differentially expressed genes (DEGs) involved in tanshinones, GA biosynthetic pathway and signaling pathway. DEGs demonstrated significantly differential expression (FDR-adjusted $P$-value $<0.05$, |fold-change| [?] 2). The average FPKM ( $\log 10$ scale) of each gene is shown in the heat map.

Figure 8. Model for SmGRAS5 regulates the GA-mediated accumulation of tanshinones in S. miltiorrhiza . GA could induce SmGRAS5response and promote the accumulation of tanshinones. SmGRAS5 could inhibit GA biosynthetic, and catalyze the precursor GGPP to synthesize more tanshinones through directly binding to the promoter ofSmKSL1 . 


\section{Supporting information}

Table S1. Primers used for qRT-PCR.

Table S2. Primers used for vector construction and positive hairy roots line selection.

Figure S1. Phylogenetic tree of SmGRAS5 transcription factor. The phylogenetic tree was constructed by the maximum-likelihood method of MEGA 6.0. Members in the same sub-branch were marked by a circle filled with the same color.

Figure S2. Transactivation activity of SmGRAS5. Yeast AH109 containing $p$ GBKT7-53+pGADT7-T (positive control), $p G B K T 7$ (negative control) and SmGRAS5-pGBKT7 were spotted onto SD/-Trp and SD/Trp-His-Ade medium, respectively.

Figure S3. Verification of positive hairy roots lines by using PCR. PCR screening of SmGRAS5 overexpressing or antisense expressing lines.

Figure S4. Growth phenotypes of SmGRAS5 transgenic hairy roots lines.

Figure S5. GA induces SmGRAS5 responses.

(A) Relative quantitative analyzes the expression of SmGRAS5 with $100 \mu \mathrm{M}$ GA treatment for $2 / 24 \mathrm{~h}$. (B) Promoter analysis of the GA response element of SmGRAS5.

Figure S6. Y1H assay of SmGRAS5 and SmCPS1 promoter.SmCPS1 promoter+pGADTr as the negative control and SmCPS1 promoter+ $p$ GADT7-SmERF6 as the positive control.

Figure S7. Purified MBP protein and SmGRAS5 protein with MBP label were verified via Western blot. M: protein marker.

Figure S8. EMSA analysis of the interaction of SmGRAS5 with the promoter of GA biosynthesis genes.

(A) EMSA analysis of the interaction of SmGRAS5 with CPS5 promoter. (B) EMSA analysis of the interaction of SmGRAS5 with KS promoter. (C) EMSA analysis of the interaction of SmGRAS5 with GA3ox1 promoter.

Figure S9. Validating the different expression levels of the identified genes from RNA-seq data by qRT-PCR. Expression of the representative GA-responsive genes in the RNA-seq experiments of G5O14. The expression of genes in the untreated ATCC hairy roots lines was set to 1. Standard errors were calculated from three sets of biological replicates. Significant differences using one-way ANOVA and S-N-K comparison tested, $P$ $<0.05$. 
Figure 1

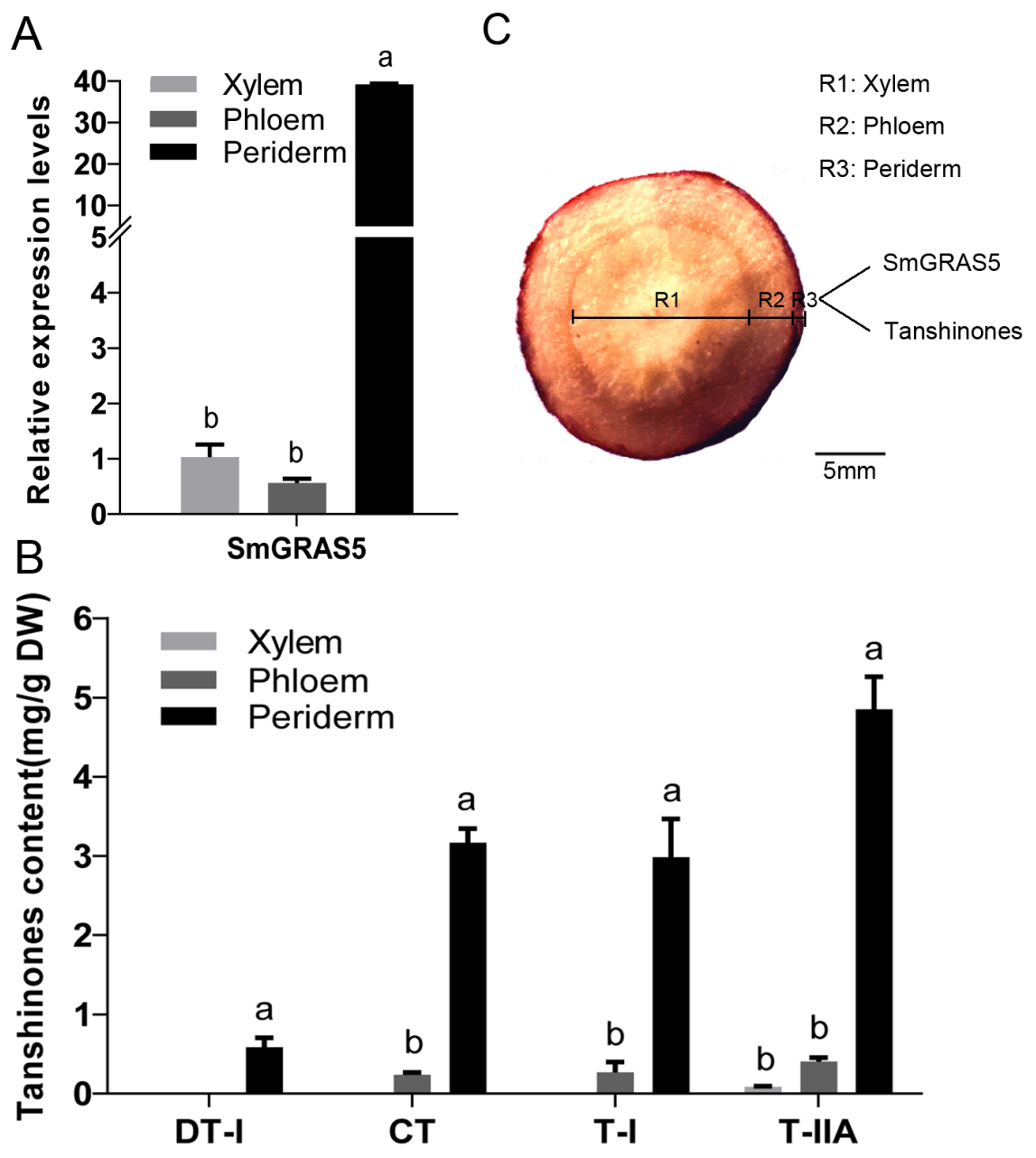


Figure 2

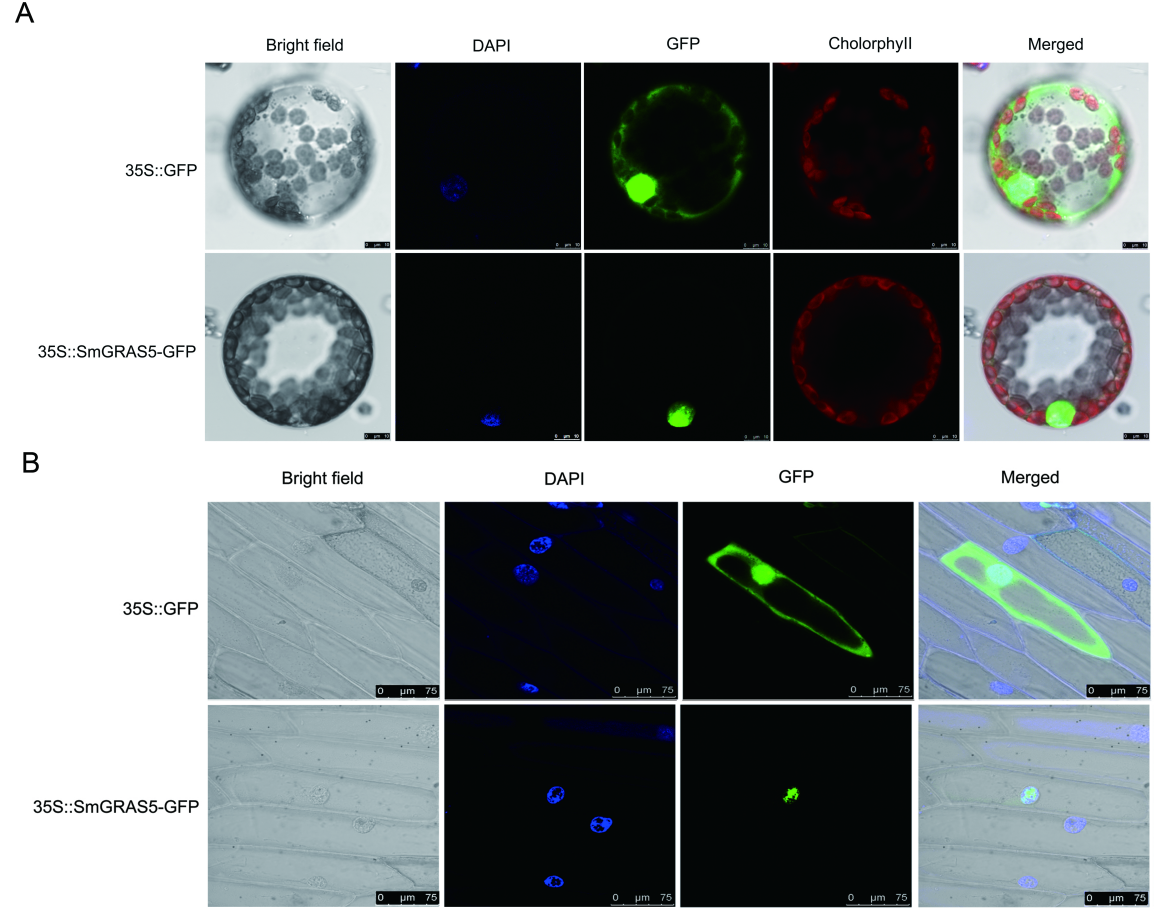


Figure 3

A

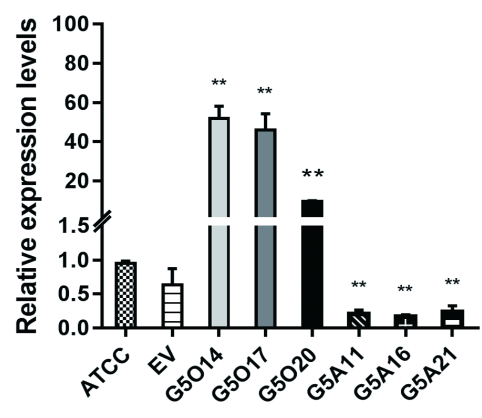

D

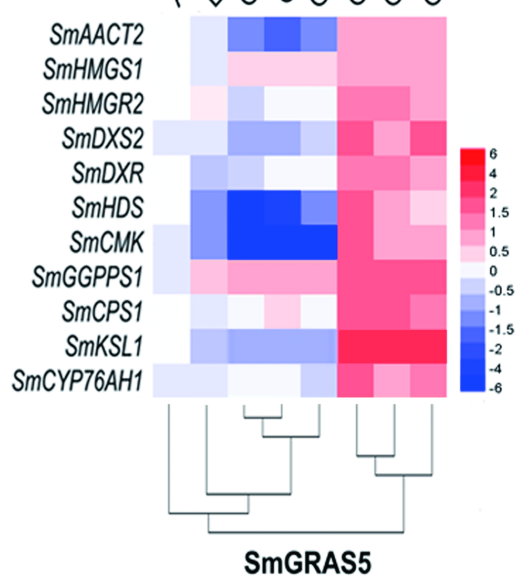

B

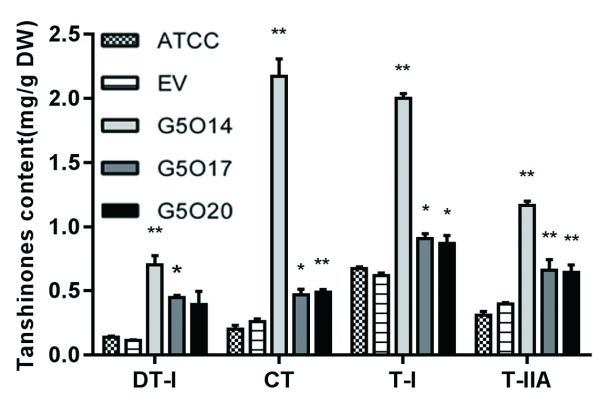

C
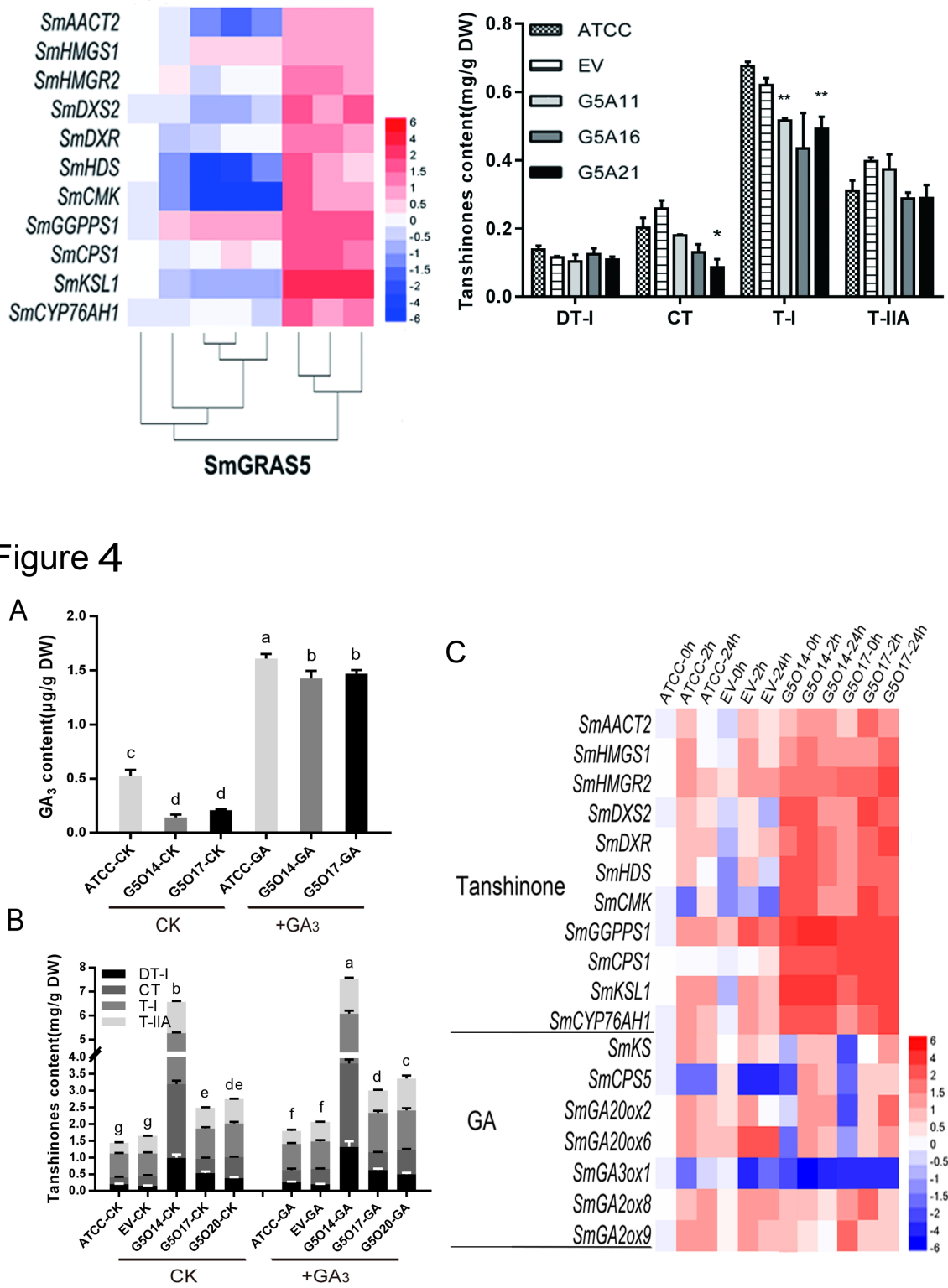

C

SMAACT2

SMHMGS1

SMHMGR2

SmDXS2

SMDXR

SmHDS

SmCMK

SMGGPPS1

SMCPS1

SMKSL1

SMCYP76AH1

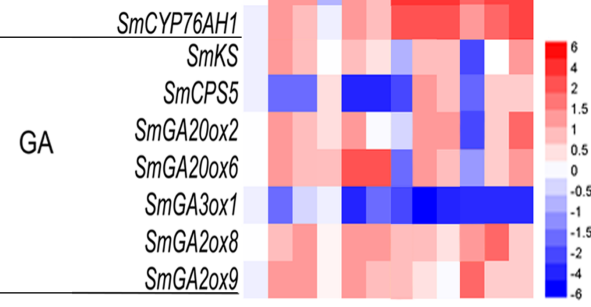

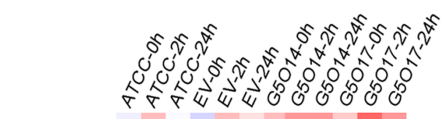


Figure 5

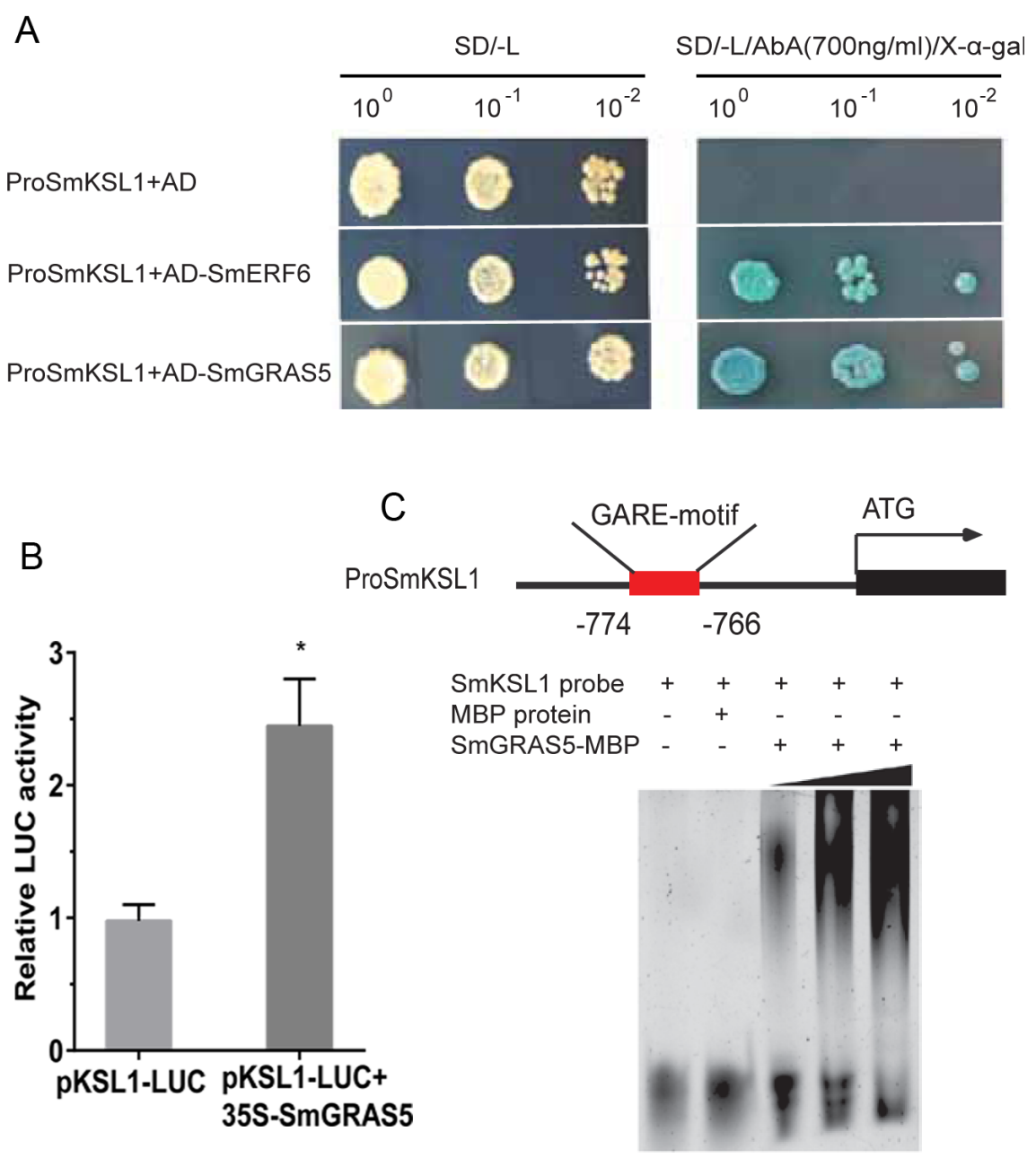


Figure 6

A

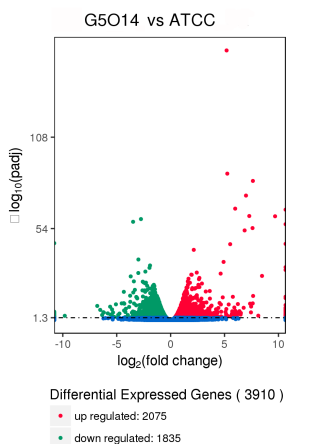

D

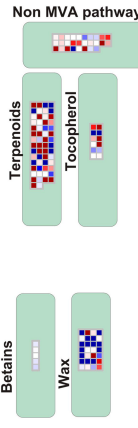

Shikimate pathway

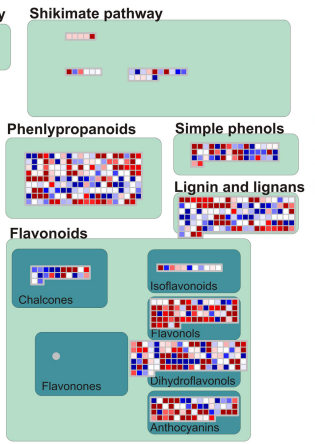

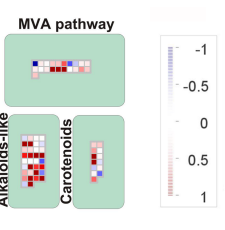

Glucosinolates Ax+m: Cyanogenic glycosides Sulfur containing

C

Statistics of Pathway Enrichment
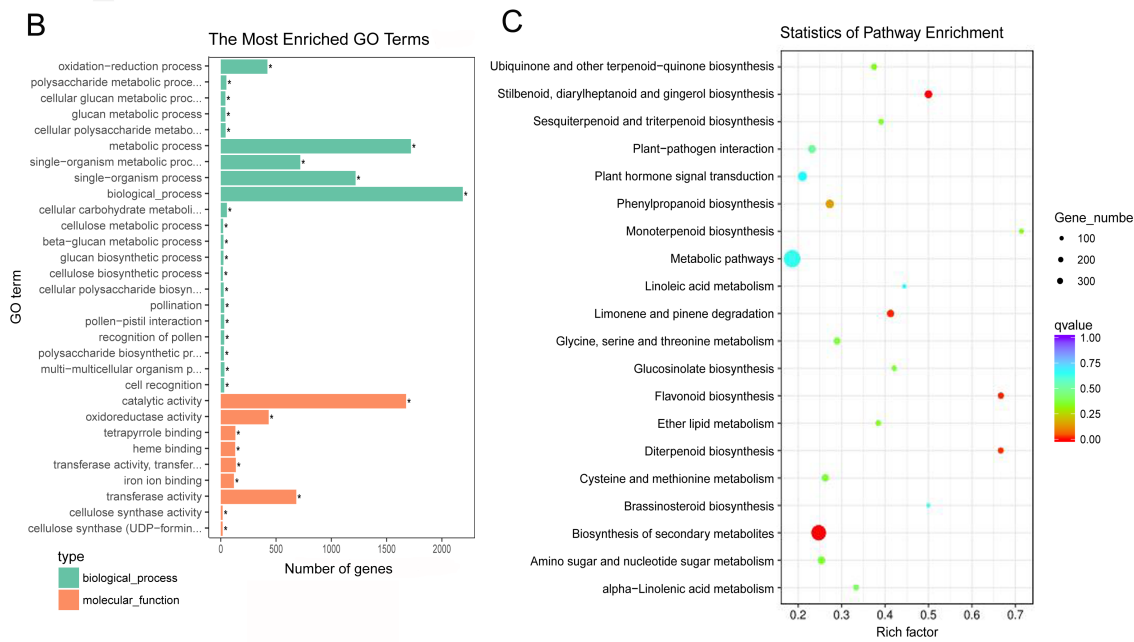
Figure 7

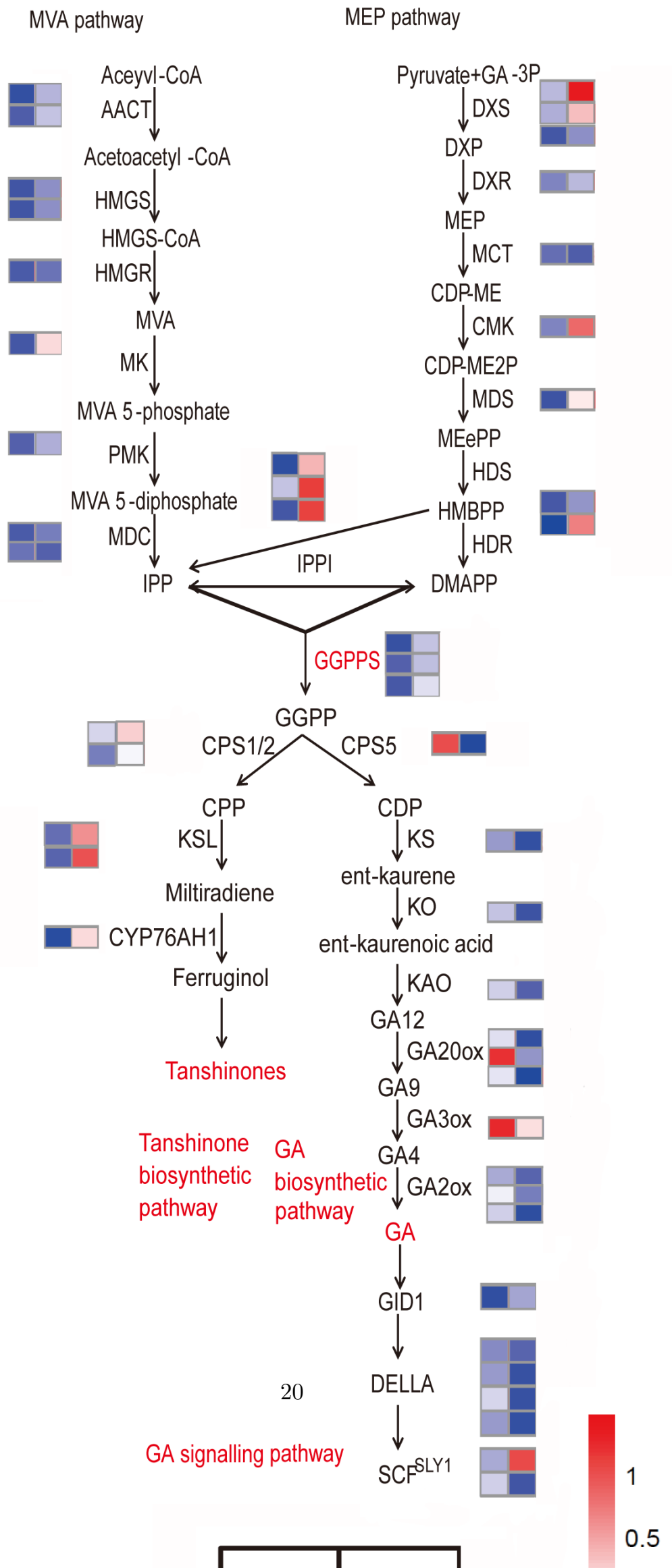


Figure 8

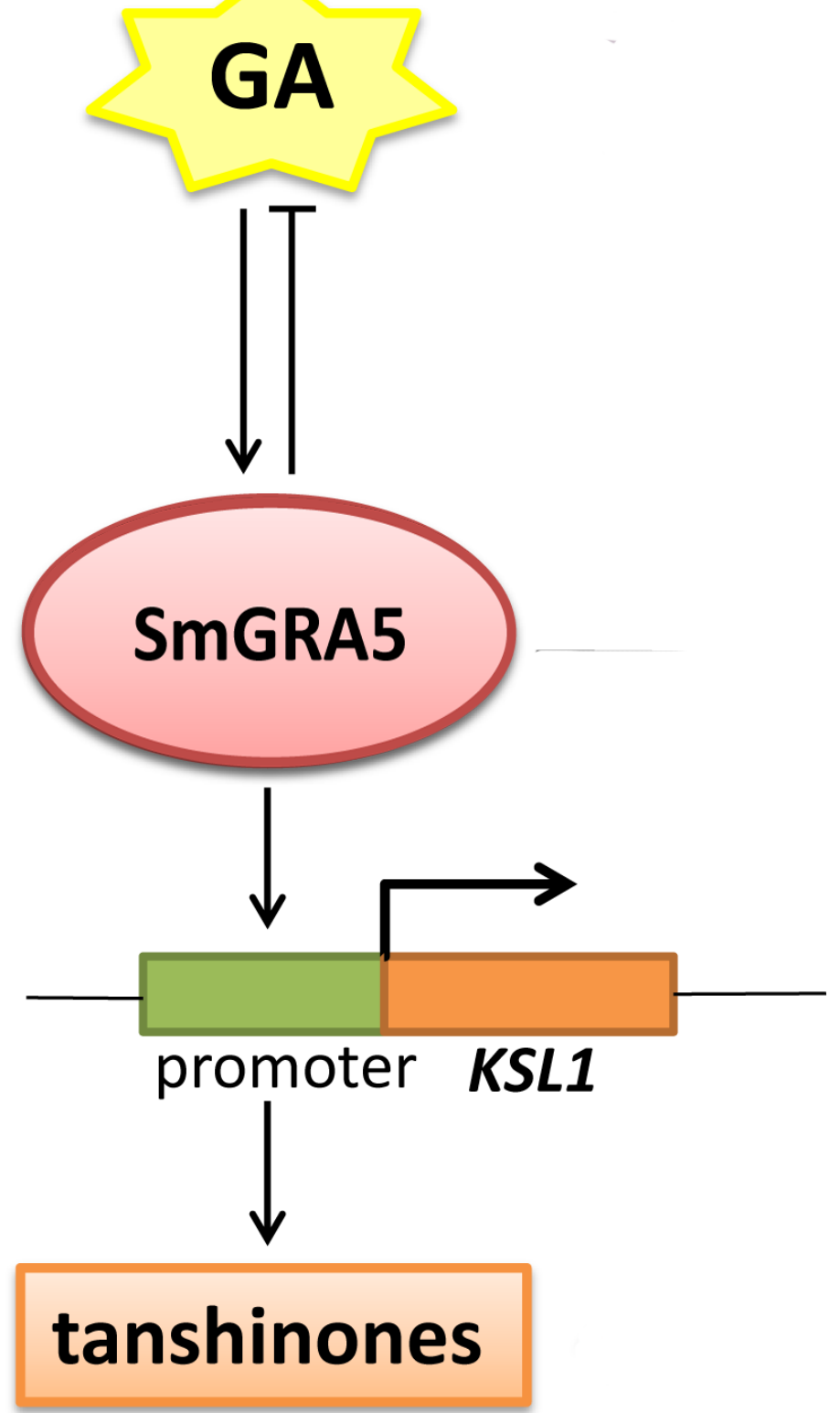

